\title{
Las voces de los conocedores y conocedoras de los pueblos originarios en la formación docente
}

\author{
Lucy Trapnell
}

Consultora independiente, miembro de Foro Educativo tsirapa17@gmail.com

RESUMEN

A lo largo de las últimas décadas se ha venido planteando la necesidad de problematizar la manera como se construye el conocimiento $y$ de poner en evidencia las relaciones entre conocimiento y poder. Una valiosa innovación, que busca abrir la educación superior a la inclusión de nuevos actores y nuevas voces, ha sido la redefinición del equipo formador de algunos institutos superiores pedagógicos y universidades convencionales para incluir conocedores y conocedoras de los pueblos originarios. No obstante, en este artículo argumento que su participación en los procesos de formación docente no necesariamente garantiza el desarrollo de prácticas que hagan evidente la existencia de formas de pensar alternativas al conocimiento hegemónico ni las múltiples formas como se producen. Para que esto ocurra, es necesario tomar conciencia de la compleja relación entre conocimiento y poder, y analizar la forma como esta se expresa en la formación superior y, de manera concreta, en la institución formadora. Sustento este argumento en la experiencia del Programa de Formación de Maestros Bilingües de la Amazonía Peruana (Formabiap), al cual he acompañado a lo largo de los últimos veintinueve años, en mi experiencia directa y en sistematizaciones y evaluaciones internas y externas del programa.

Palabras clave: conocimiento, poder, interculturalidad crítica, justicia epistémica, formación docente, Amazonía. 


\section{The voices of Indigenous Peoples' Elders in teacher Training}

\section{ABSTRACT}

During the last decades the need to question the way in which knowledge is constructed as well as its relation with power issues has come forward. An important innovation in some teacher training colleges and conventional universities is the redefinition of the teaching staff. They have included indigenous elders as an attempt to open higher education to the inclusion of new actors and new voices. However, in this article I argue that the participation of indigenous elders in teacher training processes, does not necessarily guarantee the development of practises that will highlight the existence of ways of thinking alternative to hegemonic knowledge nor the multiple ways in which knowledge is produced. For this to happen consciousness must be gained regarding the complex relations between knowledge and power, and the way in which it is expressed in higher education in general and in specific academic spaces. Drawing from the experience of the Teacher Training Programme of the Peruvian Amazon (Formabiap), which I have accompanied during the last 29 years, I sustain my argument with information gained through my direct experience with the Programme and from documents, studies and internal and external evaluations of its process.

Keywords: knowledge and power, critical interculturalism, epistemic justice, teacher training, Amazonia. 


\section{INTRODUCCIÓN}

A lo largo de poco más de una década, en América Latina se han realizado propuestas de educación superior, diferentes a las de las universidades convencionales, que buscan responder a la diversidad cultural y lingüística que caracteriza a los distintos países de la región. Entre ellas están las universidades indígenas, las universidades interculturales, las universidades interculturales indígenas y las universidades comunitarias.

En la creación de las universidades interculturales han confluido varios factores. Muchas han sido fundadas a iniciativa de dirigencias y organizaciones indígenas o afrodescendientes; algunas han sido promovidas por el Estado, como ha sucedido en México, y, en el caso del Perú, han sido resultado de iniciativas de las organizaciones de la sociedad civil y de autoridades locales y regionales.

De manera coincidente, a lo largo de las últimas décadas se ha generado mayor conciencia sobre la necesidad de problematizar la manera como se construye el conocimiento y de poner en evidencia las relaciones entre conocimiento y poder (López Soria, 2012; Santos, 2008; Walsh, 2001; Mignolo, 2000; Quijano, 2000). Unesco se ha unido a esta exigencia al plantear la necesidad de avanzar hacia una justicia cognitiva y epistémica por medio de la redistribución de oportunidades de aprendizaje, el reconocimiento y la legitimación de la cultura y conocimiento indígenas y su inclusión en la política pública (Unesco, 2017).

Sin embargo, optar por la justicia epistémica en la educación superior no es fácil: demanda un conjunto de transformaciones, tales como marcos legales más sensibles a la diversidad, nuevos tipos de certificación, y planes de estudio innovadores que consideren saberes indígenas y sus formas de transmitirlos y aprenderlos, así como nuevos criterios para la selección de las y los formadores, con el fin de asegurar que, además de contar con doctores y magíster, se pueda garantizar la presencia de conocedores y conocedoras de los pueblos originarios, además de la creación de mecanismos de gestión participativa. 
Los aspectos relacionados con la innovación del plan de estudios y la inclusión de conocedores y conocedoras de los pueblos originarios han sido abordados por algunos programas de formación docente en la especialidad de educación intercultural bilingüe (EIB), desarrollados por institutos superiores pedagógicos del Estado y de universidades convencionales. Algunas instituciones dedicadas a formar promotores de salud también han realizado experiencias de innovación curricular para incluir saberes de los pueblos originarios. Sin embargo, la Universidad Nacional Intercultural de la Amazonía Peruana (UNIA), creada el año 1999, tiene escasos avances con relación a estos temas

Una valiosa innovación en la línea de incluir nuevos actores en la educación superior ha sido la redefinición del equipo formador de algunos institutos superiores pedagógicos y universidades, que han incorporado conocedores y conocedoras de los pueblos originarios de manera permanente o eventual. No obstante, en este artículo argumento que la participación de estos conocedores en los procesos de formación docente no necesariamente garantiza el objetivo de una educación intercultural crítica, orientada a explicitar la existencia de formas de pensar alternativas al conocimiento hegemónico, así como las múltiples formas como se producen. Para que esto ocurra, es necesario tomar conciencia de la compleja relación entre poder y saber, y analizar la manera como esta se expresa en la formación superior y, de manera concreta, en la institución formadora. Esto no es nada fácil, porque implica tomar distancia frente a un conjunto de temas poco cuestionados entre los docentes formadores, como la jerarquía de la ciencia y el conocimiento disciplinar, en los que precisamente sustentan su actividad y prestigio académico. Además, requiere comprender y aceptar que existen otras formas de entender la realidad, el conocimiento y el aprendizaje, así como la manera como se construye y transmite el conocimiento.

Sustento este argumento a partir de la descripción y análisis del proceso que vivió el Programa de Formación de Docentes Bilingües de la Amazonía Peruana, programa coejecutado, desde 1988, por la confederación indígena Aidesep y el Instituto Superior Pedagógico Loreto, que fue la primera institución formadora del Perú en incluir la presencia de conocedores de los pueblos originarios en su equipo de formadores. Me baso en mi conocimiento del programa, primero como miembro del equipo fundador y codirectora, y luego como docente y asesora, así como en las evaluaciones y sistematizaciones de esta experiencia. 


\section{MARCO CONCEPTUAL}

Empezando por una simple definición, se podría señalar que la educación intercultural considera la diversidad cultural como uno de los pilares fundamentales de una propuesta educativa. En tal sentido, sitúa la existencia de grupos humanos de distintas herencias culturales en el foco de su práctica.

Sin embargo, la manera en que se concibe la diversidad cultural y cómo se concreta la educación intercultural depende en gran medida del contexto sociopolítico en el cual se desarrolla y del enfoque de interculturalidad en el que se inscribe. De esta manera, no existe una visión compartida de la educación intercultural que vaya más allá de la definición básica antes planteada.

Existen distintas miradas a la educación intercultural. Desde una mirada que responde a lo que Fornet Betancourt (2001) y Tubino (2004) denominan interculturalidad funcional, la educación intercultural está básicamente orientada a promover el diálogo, la convivencia y la tolerancia. Si bien reconoce la diversidad, su meta es incluirla dentro del orden social establecido sin cuestionar las causas que generan la asimetría y desigualdades sociales, económicas y culturales; en tal sentido, es funcional al sistema neoliberal imperante. En el campo curricular, ofrece posibilidades para la inclusión de algunos saberes indígenas sin cuestionar la jerarquía del conocimiento hegemónico. En el plano escolar, su visión de la diversidad se reduce a lo folclórico, fundamentalmente centrado en visibilizar la cultura material.

Desde el enfoque de interculturalidad crítica, se plantea la necesidad de ubicar el discurso sobre la diversidad en una discusión más amplia que abarque la dimensión económica y política. En este sentido, la interculturalidad crítica no solo busca respetar y valorar la diversidad cultural y lingüística y generar procesos de diálogo entre personas procedentes de diferentes tradiciones culturales, sino que apela a «cambios profundos en todas las esferas de la sociedad y aporta a la construcción de sociedades alternativas que refundan el Estado nacional en América Latina y otras partes del mundo» (Ávila, 2014, p. 42).

Quijano (2000), Mignolo (2000) y otros sociólogos dedicados a los estudios postcoloniales aportan al debate sobre el concepto de interculturalidad al utilizar la noción de «diferencia colonial», la cual sitúa el debate en el problema estructural colonial - racial que ayuda a entender desde dónde se construye la diferencia. Según estos autores, la diferencia étnico-cultural es una construcción social, es decir, algo que no es innato, que no siempre ha existido de la manera como hoy lo conocemos. En efecto, durante la Colonia los conquistadores clasificaron, 
nombraron y jerarquizaron a los grupos sociales. En este proceso elaboraron la distinción entre seres superiores e inferiores, y en particular, entre aquellos que producían riqueza y aquellos que vivían del trabajo de otros. Desde entonces, y a lo largo de la historia, se fue instaurando un imaginario social que justificaba y garantizaba este orden jerárquico. A ello contribuyeron la teoría evolucionista, las crónicas y relatos de viajeros y misioneros, los medios de comunicación, así como la escuela y sus materiales. Esta noción de la existencia de seres superiores y seres inferiores está en la base de las autopercepciones y de las percepciones sobre «el otro».

Los estudios postcoloniales han sido, además, uno de los marcos de referencia de la conceptualización de la interculturalidad crítica en el campo epistémico. Desde una aproximación que aborda la estrecha relación existente entre los patrones de poder económico y político y la producción discursiva, los estudios postcoloniales cuestionan la idea de un saber deslocalizado y plantean la existencia de centros de poder en los que se genera un saber que se impone y se naturaliza (Quijano, 2000).

Como señala Ávila (2014), este modelo de conocimiento, construido desde centros de poder que justamente coinciden con los centros de poder económico, rechaza la validez de los saberes del otro, de todos los otros, y les niega el derecho a sus propias opciones culturales, modos y vida y, con frecuencia, a la vida misma. En esto precisamente consiste la colonialidad del saber que valida una determinada visión del mundo que produce «verdades» que llegan a naturalizarse a tal extremo que se ven como «normales» y «objetivas», y que se instalan en el «sentido común» de las personas para legitimar la desigualdad y la jerarquización social.

Esta aproximación enriquece el debate sobre la interculturalidad al poner el tema del conocimiento en el centro de las relaciones de poder, en la medida en que, como señala Walsh (2001), «ayuda a estructurar, legitimar y justificar el poder dominante y la subalternidad».

Los estudios postcoloniales intentan visibilizar conocimientos diferentes de los hegemónicos, reconociendo su aporte desde distintas lógicas y formas de pensar el mundo.

Como señala Santos (2008), reivindicamos una ecología de los saberes donde no se pretende desacreditar a las ciencias o volverse anticientíficos, sino que el saber científico pueda y pretenda dialogar con el saber laico, el saber popular, el saber indígena. Lo importante no es ver cómo el conocimiento representa lo real, sino conocer lo que un determinado conocimiento produce 
en la realidad. Además, es importante asumir —en pos de un proyecto civilizatorio y utópico posible - que hay muchas maneras de entender el mundo y que la diversidad epistémica debe ser un componente central sobre el cual camine el enfoque educativo intercultural que desarrollan nuestras instituciones educativas (Ávila, 2014, p. 41).

\section{EL PUNTO DE PARTIDA DE FORMABIAP}

El Programa Formabiap se desarrolla desde 1988 en la ciudad de Iquitos, capital de la región Loreto, en el marco de una coejecución entre el Instituto Superior Pedagógico Loreto y la confederación nacional indígena Aidesep.

Al ser un programa de formación docente coejecutado por una confederación indígena, Formabiap asumió el objetivo de formar maestros capaces de poner en práctica un nuevo tipo de educación que promueva la revalorización del modelo social de su pueblo y potencie la participación de los niños y niñas como actores y miembros de este, en el marco de un Estado pluricultural (ISPL-Aidesep, 1997). En coherencia con este propósito, Formabiap se planteó la necesidad de que sus estudiantes asumiesen una mirada crítica frente al papel asimilador que ha cumplido la escuela en sus pueblos y desarrollen una nueva visión sobre ella y su papel como educadores indígenas. Desde la perspectiva de una escuela «desde y para los pueblos indígenas amazónicos», el programa se orientó a preparar docentes capaces de formar a las nuevas generaciones de su pueblo originario con un fuerte sentido de identidad y valoración de su herencia cultural y poseedores de un conjunto de capacidades que les permitan desenvolverse en su medio y en otros espacios.

Esto implicó cambios sustanciales en el currículo de formación docente vigente en ese entonces (Trapnell, 2009). Desde su creación, el programa puso un fuerte acento en la necesidad de incorporar saberes indígenas en su propuesta curricular. Para ello adoptó las siguientes medidas:

a. Diseño de un plan de estudios innovador que fue enriqueciéndose a medida que el equipo formador se fortalecía y potenciaba su conocimiento sobre los pueblos originarios atendidos por el programa.

b. Inclusión de conocedores indígenas en su plana docente, con el fin de que ayuden a los estudiantes a profundizar en el manejo de la cultura y la lengua 


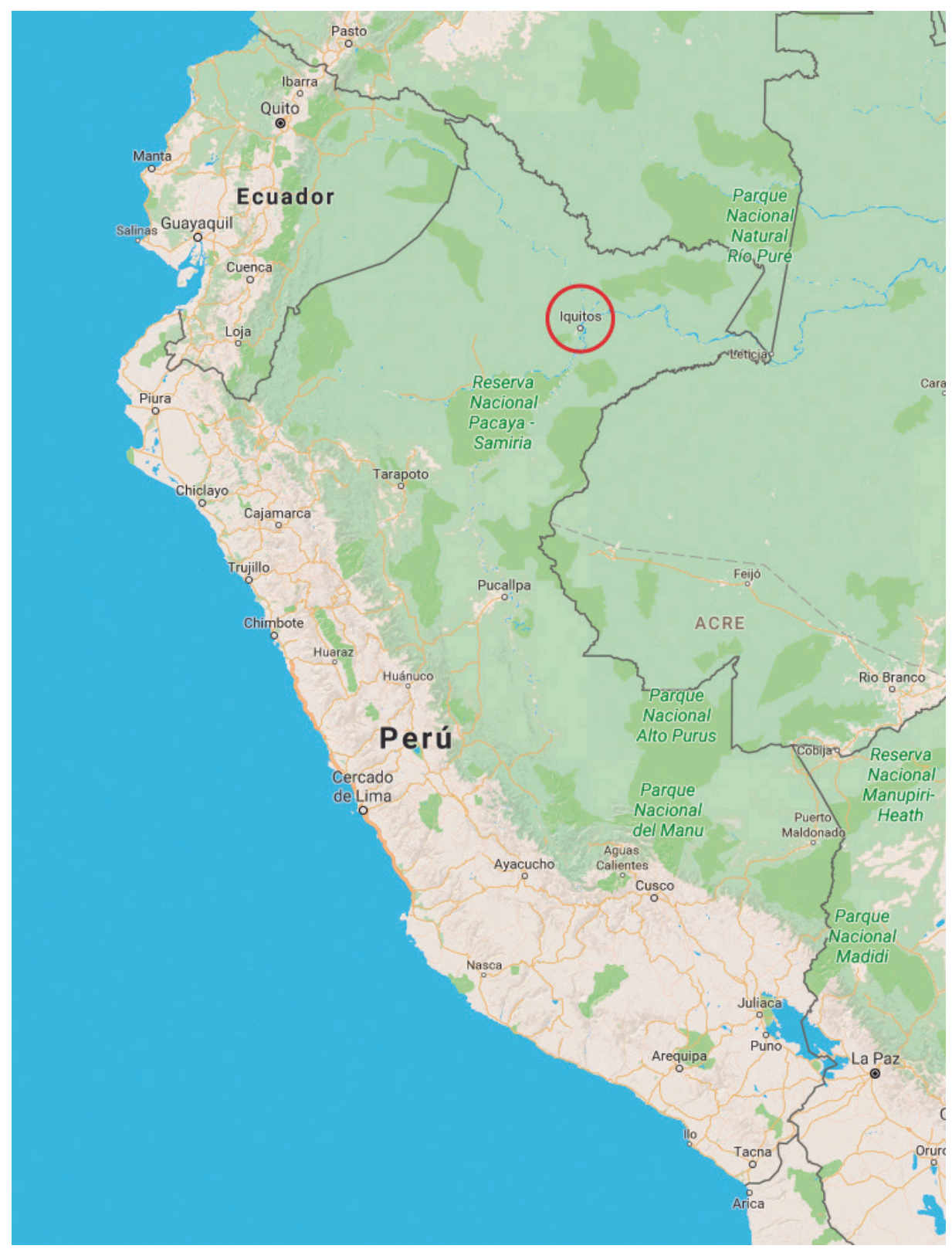

Mapa del Perú con incidencia en Iquitos, región Loreto. 
de sus pueblos y a desarrollar capacidades orales en ellas, en el caso de pueblos donde la lengua ya no se usa de manera cotidiana.

c. Organización del año académico en ciclos presenciales en Iquitos y no presenciales en comunidades del pueblo de origen de los estudiantes, con el fin de que tuvieran espacios para profundizar los saberes de su pueblo en las comunidades y con el apoyo de los conocedores que los habían apoyado durante el ciclo presencial.

Los primeros conocedores de los pueblos originarios, entonces llamados especialistas, fueron siete varones de los pueblos ashaninka, awajún, shipibo, kukama, wampis, witoto y bora. Seis de ellos solo habían cursado los primeros años de educación primaria y uno era un maestro cesante. Todos tenían más de cincuenta años y habían sido elegidos por sus federaciones. Ellos fueron contratados con fondos de la cooperación internacional. Luego se sumaron conocedores de otros pueblos, siempre varones, hasta llegar a quince. La inclusión de mujeres recién se dio a inicios de la década de 2000 y tuvo mucho que ver con la manera como cambió el papel de los conocedores en el programa, según explicaré más adelante.

Durante los ciclos presenciales en Iquitos, los conocedores participaban en las clases a cargo de los docentes de las diferentes áreas, particularmente Educación, Sociedad, Naturaleza y Lenguaje. Ahí contribuían con sus saberes cuando los docentes les pedían información sobre algún aspecto relacionado con su herencia cultural. Además, participaban en talleres de lengua originaria, enseñaban la lengua a los miembros de su equipo local ${ }^{1}$ y sistematizaban algunos temas con apoyo de los docentes asignados a su equipo. También iban al campo durante los ciclos no presenciales con el fin de apoyar a los estudiantes en el desarrollo de sus trabajos de profundización de la herencia cultural de su pueblo.

Esta modalidad de participación de los conocedores en el proceso formativo durante los primeros catorce años de vida de Formabiap respondía a la manera como el programa abordaba la inclusión de saberes indígenas en su plan de estudios y a su manera de entender la relación entre conocimiento y poder, como explico a continuación.

El programa está estructurado en equipos locales organizados por pueblos indígenas o por pueblos con una tradición cultural similar. En cada uno participan estudiantes, conocedores y docentes. Hasta mediados de la década de 1990, cada equipo contaba con un lingüista o antropólogo. 


\section{La relación entre conocimiento y poder}

Si bien Formabiap hizo un gran esfuerzo por incorporar los saberes indígenas en su propuesta curricular, la manera de hacerlo estuvo condicionada por cómo se abordaba la relación entre el conocimiento indígena y la ciencia. Su equipo fundador asumía que el manejo del aparato conceptual y metodológico de las diferentes ciencias ayudaría a empoderar a los futuros maestros indígenas, en la medida en que les daría mayor seguridad y confianza y les brindaría instrumentos para analizar y cuestionar los procesos de subordinación y marginalización de sus pueblos (Trapnell, 2009).

Sobre la base de una reflexión sobre las respuestas de «confianza ciega» o «resistencia pasiva» que los comuneros indígenas tienen frente a la «palabra del ingeniero», al cual consideran dueño de un conocimiento de calidad superior que no puede ser abiertamente contradicho, se argumentaba lo siguiente:

Para el alumno, el método científico debe devenir en el instrumento de acercamiento a la realidad mediante el cual él formula su propia opinión, una opinión independiente que puede expresar y afirmar frente a los que hacen valer su palabra en base de su estatus social y con el cual él evalúa críticamente las afirmaciones que quieren imponerse por la gracia del estatus. El método científico le da los criterios para expresar sus exigencias frente al discurso dominante, para cuestionarlo y obligar al interlocutor, que se reposa sobre el poder del estatus de su palabra, a adoptar la posición modesta común de la razón humana frente a la realidad objetiva, perceptible, observable, descriptible y explicable (notas de preparación del curso de Antropología. Jorge Gasché, 1989).

Esta opción por una formación científica coincidía con las aspiraciones de los dirigentes de Aidesep, quienes ponían un fuerte énfasis en la necesidad de formar maestros en los conocimientos de las disciplinas científicas, sin dejar de lado sus propios conocimientos (Nukguag, 1985).

Estos supuestos fueron incorporados en los dos primeros lineamientos curriculares del programa y se mantuvieron hasta inicios de la década de 2000. No obstante, a lo largo de la década de 1990, se dio, en las diferentes áreas de formación, cada vez mayor cabida a los saberes y formas de entender la realidad de los pueblos originarios. A lo largo de este proceso, se abordó su manera de entender el medio social y natural y sus concepciones sobre el territorio, la persona y el aprendizaje, entre otros saberes que buscaban enriquecer la propuesta curricular desde un enfoque intercultural. 


\section{EL PUNTO DE QUIEBRE}

Si bien el trabajo que venía realizando Formabiap definitivamente fue un gran avance con relación al poco peso que tenían los conocimientos indígenas en los procesos de formación docente en otras instituciones que ofrecían la especialidad EIB, a inicios de la década de 2000 se dio un conjunto de situaciones que llevaron al programa a revisar la manera como estaba abordando el vínculo entre los saberes indígenas y la ciencia occidental.

Una de esas situaciones tuvo que ver con las demandas de algunos conocedores indígenas. Luego de varios años de trabajo en Formabiap, uno de ellos empezó a cuestionar su rol durante el ciclo presencial en la comunidad educativa de Zungarococha, sede de Formabiap desde mediados de la década de 1990. Su malestar inicialmente se expresó con relación al poco tiempo que se les daba para aportar en las clases. En efecto, la presencia de varios sabios para estudiantes de por lo menos cinco pueblos originarios les ofrecía muy poco tiempo para intervenir. Sin embargo, el problema era más profundo y estaba básicamente referido al tipo de producción discursiva que se privilegiaba en el desarrollo de las clases y, de manera particular, en el área de Naturaleza. El conocedor ashaninka Eusebio Laos planteó este tema en varias oportunidades. Por razones de espacio me limitaré solo a uno de sus cuestionamientos.

Al término de una clase del área de Naturaleza acerca de cadenas alimentarias, don Eusebio se quejó por el excesivo tiempo dedicado a conversar sobre dicho concepto y comentó: «Eso de quién come a quién lo sabe cualquier niño. Pero eso a quién le interesa. Eso no es lo verdaderamente importante». Como luego precisó, lo que verdaderamente importa son las relaciones de cooperación que se establecen en la naturaleza y cómo ellas les enseñan a los hombres a actuar. En este sentido, si bien reconocía la existencia de un conjunto de vínculos entre animales, plantas y seres humanos, le importaban más los de tipo social que los estrictamente biológicos. El especialista ashaninka ofreció mayores alcances sobre su punto de vista en el siguiente texto extraído de la exposición «Cuando las aves eran personas» ${ }^{2}$ :

Las codornices andan juntas, juntas corren, juntas regresan. Si una de ellas es asaltada, todas van a defender a su hermana, hermano, padre o madre. Ellas son muy prevenidas. También saben compartir. Cuando una encuentra una

2 «Cuando las aves eran personas» fue parte de la exposición «Amazonía al descubierto», presentada en el Museo de Arte del Centro Cultural de San Marcos entre octubre y diciembre de 2005 . 
lombriz le da la mitad a la otra. Los ashaninka observamos a esta ave y la imitamos por su forma de ser, porque tiene buen espíritu, es dadivosa. No es como otras aves, como la chicua, el gallinazo y el pajarito mari, que paran maldiciendo y son de mal agüero.

De igual manera, a don Eusebio le preocupaba la falta de un discurso sobre el respeto que debía existir entre las personas y los seres que las rodean. Sostenía que el discurso del área de Naturaleza estaba más centrado en la protección, el equilibrio, el cuidado y el manejo, y que dejaba de lado lo más importante, que era el respeto.

Los cuestionamientos de Eusebio Laos estaban sistemáticamente referidos al área de Naturaleza, y eso tenía que ver con su crítica a la manera como se organizaba el discurso del área desde la lógica discursiva de la Ecología. Esta opción se expresa claramente en la fundamentación del área, en la cual los conocimientos indígenas se explicitan y abordan desde el marco interpretativo de la ciencia.

La formación que recibe el futuro maestro en el área de Naturaleza conlleva dos procesos funcionalmente ligados: por un lado, la explicitación de conocimientos indígenas referentes a la naturaleza y el manejo del bosque [...] y por otro, la implementación, a través de los conceptos de la ecología, de conocimientos de las ciencias naturales y otras disciplinas auxiliares, de un entendimiento sistemático de la naturaleza en general y del bosque amazónico en particular, y del ser humano como organismo constitutivo y responsable de este. El segundo proceso contiene los instrumentos (el metalenguaje) para la realización del primero, que procede mediante el método inductivo intercultural (motivación, verbalización/objetivización y sistematización/interpretación) [...] Con esto, [...] se apunta a que el futuro maestro adquiera instrumentos conceptuales y un método que le permita profundizar en el futuro nuevos aspectos. La manera como Formabiap había planteado la relación entre los saberes indígenas y la ciencia también fue cuestionada en una evaluación realizada por Guzmán y Monroe (2003). Los evaluadores señalaron que el programa había recurrido a la traducción, al interpretar el conocimiento indígena con los términos del conocimiento científico, a través de una rejilla conceptual moderna pretendidamente universal. Además, hicieron hincapié en la falta de atención de Formabiap a las categorías y procesos de los temas tratados y que él pueda transferir a otros campos de la realidad indígena en el transcurso de la aplicación del currículo de primaria, mediante nuevas investigaciones-aprendizaje que llevará a cabo en el ejercicio de su profesión (ISPL-AIDESEP, 1997, p. 59).

En respuesta a los temas planteados en la evaluación, en abril de 2004 el equipo docente de Formabiap organizó un conversatorio interno denominado 
«Interculturalidad, diferencia colonial y descolonización del saber». En él también participó el vicepresidente de la confederación indígena Aidesep. La primera parte del conversatorio estuvo orientada a reflexionar sobre los múltiples significados del concepto de interculturalidad y la manera como lo entiende el programa. Se discutió la conveniencia de incorporar el concepto de «diferencia colonial» en el enfoque de interculturalidad del programa, con el fin de poner énfasis en el hecho de que la diferencia étnica cultural es una construcción social basada en la distinción entre seres superiores y seres inferiores, la cual ha sido consustancial a la empresa colonial y está en la base de las autopercepciones y percepciones del «otro». Se reflexionó sobre el hecho de que, si bien es importante asumir una posición de respeto, afirmación y valoración de la diversidad cultural y lingüística, y de afirmación de los derechos colectivos indígenas, es necesario entender que el desarrollo de actitudes favorables a la diversidad no es un tema de manejo de información y de buenas intenciones, sino que tiene que ver con cambios sustanciales en los ámbitos económico, social y político que se han desarrollado en el país y la región, a partir de la afirmación de una diferencia étnico-cultural jerarquizada.

A lo largo de este conversatorio, se desarrolló un proceso de reflexión y evaluación crítica de la incidencia que ha tenido la ciencia occidental en la organización de los currículos de formación docente y de educación primaria de Formabiap. Se discutió sobre el hecho de que, aun cuando el programa ha tenido entre sus objetivos principales la formación de un discurso crítico y liberador, y le ha dado un significativo peso al tratamiento de los conocimientos indígenas como no lo había hecho ningún programa EIB hasta entonces en el Perú, haber utilizado la ciencia como un medio para interpretarlos y validarlos ayudaba a enfatizar la superioridad de esta. Sin embargo, también se recordó que, en sus inicios, el programa apeló a este conocimiento en la medida en que asumía que su manejo ofrecería a los futuros maestros la posibilidad de analizar la realidad y cuestionar las diferentes maneras en las que se manifiesta la dominación, así como buscar alternativas frente a ellas.

Retomando los temas tratados en la evaluación realizada por Guzmán y Monroe a fines de 2003, se debatió sobre el peligro de caer en procesos involuntarios de asimilación y de subvaloración de los conocimientos provenientes de tradiciones culturales no hegemónicas

La última parte del taller estuvo orientada al análisis de la manera como Formabiap abordaba el proceso de construcción de aprendizajes y los retos que tendría que asumir desde una perspectiva de justicia epistémica. Se contempló la 
necesidad de construir otros criterios de representación de la realidad, a través de la definición de nuevos marcos conceptuales, analíticos y teóricos que apelaran a las visiones de los pueblos indígenas. En la discusión, se enfatizó que la idea no era construir un pensamiento indígena tradicional, estático y congelado, sino más bien marcos interpretativos abiertos y sujetos a permanentes reinterpretaciones.

La discusión sobre el poder llevó la reflexión más allá del tema del conocimiento y se pudo apreciar que no solo se trataba de relaciones entre conocimientos, sino entre grupos e individuos provenientes de diferentes ubicaciones sociales y tradiciones culturales y académicas (docentes indígenas, docentes no indígenas, conocedores, asesor científico). Durante el taller, varios formadores señalaron que el «poder de la ciencia» se evidenció en la relación jerarquizada que existía entre todos estos actores. Un caso que mereció especial atención fue la situación de los conocedores indígenas. Si bien es cierto que el programa planteaba la relación del sabio con los formadores no indígenas de las distintas áreas en términos de una relación horizontal de aprendizaje, se veía que su participación en clases estaba muy condicionada por el tiempo y los temas fijados por el responsable del curso.

Sin embargo, también se vio que el tema no solo tenía que ver con discursos subalternos sino con la manera como se estaba abordando el aprendizaje de los saberes indígenas en el programa. Por ello, en el transcurso de estas reuniones se planteó la necesidad de abrir espacios en el proceso formativo que permitieran abordar los saberes indígenas al desarrollo de proyectos relacionados con actividades socioproductivas (fabricación de cerámica, tejido de algodón o fibras vegetales, elaboración de tintes y de remedios vegetales, y construcción de canoas, entre otras) que se realizan en las comunidades indígenas, desde sus propias lógicas y estrategias.

\section{LAS NUEVAS PROPUESTAS}

Los conocedores y los docentes indígenas señalaron la necesidad de vincular la transmisión de saberes con la manera como se venía haciendo en la propuesta de educación primaria de Formabiap, es decir desde el desarrollo de proyectos socioproductivos.

La implementación de los proyectos generó varios cambios en la organización del trabajo al interior del programa y redefinió el papel de los docentes y conocedores sabios indígenas. Hasta entonces, los responsables de las áreas asumían la planificación y desarrollo de las labores académicas. En adelante esta responsabilidad sería compartida por los equipos locales de cada pueblo originario, 
dado que los especialistas y docentes indígenas tendrían la responsabilidad de planificar, conducir y evaluar los proyectos relacionados con la tradición cultural indígena. Esto significó un gran cambio con respecto al papel de estos equipos, que hasta ese momento habían estado dedicados básicamente al acompañamiento y tutoría de los estudiantes.

Este cambio permitió a los conocedores y docentes indígenas asumir un nuevo tipo de protagonismo en el desarrollo del programa. La opción por los proyectos también significó una nueva lógica de selección de temas de aprendizaje. Hasta entonces el equipo docente se había guiado del cartel de alcances y secuencias de los lineamientos curriculares, en los cuales los conocimientos de los pueblos indígenas se abordaban a partir de las categorías de aproximación y análisis de las diferentes áreas. El trabajo con proyectos implicaba otro tipo de acercamiento a estos saberes y exigía abordar capacidades y contenidos que no necesariamente correspondían a la lógica temporal del cartel o que no habían sido considerados en él. Sin embargo, los cambios realizados en el programa no significaron que se dejara de lado la necesidad de manejar conceptos y herramientas elaboradas por las diferentes disciplinas científicas. Por ello era necesario pensar en diferentes estrategias de formación.

A lo largo de varios años, se desarrollaron proyectos vinculados con la fabricación de canoas, de instrumentos de caza y pesca, de instrumentos musicales, tejidos de fibra, elaboración de alimentos y bebidas y de construcción de viviendas, entre otros. Los proyectos se desarrollan una vez al año a lo largo del primer semestre, en la medida que el segundo está básicamente dedicado al trabajo de investigación y la práctica preprofesional en el campo.

Las estrategias de aprendizajes incluyen el desarrollo de procesos de observación, imitación y práctica de las actividades socioproductivas, la escucha de relatos, el compartir anécdotas vinculadas con el desarrollo de la actividad y la socialización de consejos para garantizar el logro del producto. En algunos casos se han considerado purgas y dietas.

Los cambios significativos que realizó Formabiap para abordar el aprendizaje y transmisión de saberes indígenas también lo llevaron a cuestionar la falta de mujeres conocedoras. Este tema ya había sido planteado anteriormente por las estudiantes, pero no se había logrado superarlo a causa de la dificultad de contar con mujeres adultas en los momentos en que el programa las requería. Finalmente, se consideró que lo más oportuno era trabajar con parejas de esposos, cuando esto fuera posible. 
El proceso de inclusión de los proyectos en la formación docente ha implicado varios ajustes a lo largo del tiempo. Uno de ellos se refiere al horario. Inicialmente se decidió dedicarles dos mañanas continuas a la semana. Actualmente se ha optado por un día entero. Sin embargo, en los últimos años se ha tomado conciencia de que el desarrollo del proyecto no puede estar circunscrito a las horas académicas. De hecho, en muchas ocasiones los estudiantes desarrollan las actividades del proyecto desde el amanecer, con el fin de adecuarse a la manera como ellas son efectuadas en las comunidades.

Otro cambio importante tiene que ver con la manera de realizar el diálogo de saberes. Durante varios años, este estuvo restringido a la socialización de aprendizajes entre los estudiantes de diferentes pueblos al finalizar el proyecto. A partir del año 2016, se decidió incluir los aportes que ofrecen la ciencia occidental y otras tradiciones culturales, no presentes en el programa, referidos al tema que se trabaja.

A raíz de este último cambio, se ha realizado una serie de modificaciones en la estrategia de desarrollo de los proyectos y en su diseño. Actualmente, se desarrollan por fases. En cada una de ellas, se definen los saberes del pueblo que se deben profundizar, así como los saberes de otros pueblos que se socializarán y los aportes que plantean diferentes disciplinas científicas $u$ otras tradiciones culturales acerca del tema tratado, a partir de una definición clara de los propósitos del diálogo.

\section{A MANERA DE CONCLUSIÓN}

La reflexión de Formabiap sobre la relación entre conocimiento y poder y el modo como se estaba dando en el programa generó un conjunto de cambios en su vida institucional y en el proceso de formación de los futuros docentes. Uno de ellos tuvo que ver con el papel que estaban cumpliendo los sabios y la necesidad de incorporar sus prácticas discursivas, y con la manera en que se aprende y transmite el conocimiento en sus pueblos originarios.

Hoy en día, la inclusión de conocedores indígenas en los procesos de formación docente no es exclusiva de Formabiap. Los Lineamientos Curriculares de Formación Docente en la especialidad EIB, recientemente diseñados por el Ministerio de Educación, tienen un capítulo dedicado a este tema y consideran el desarrollo de proyectos socioculturales y productivos como una de las estrategias fundamentales del proceso de formación. 
Desde el año 2012, encontramos conocedores indígenas, también llamados sabios, en el programa de EIB de la Universidad Científica del Perú (Iquitos) y en el programa Nopoki de la Universidad Sede Sapientae (Atalaya). En ambas universidades cuentan con contratos a tiempo completo. La Universidad Intercultural Juan Santos Atahualpa de la Selva Central (La Merced), en proceso de creación, considera además la presencia de sabios y sabias indígenas en su equipo formador. También se observa su presencia en algunos pedagógicos del área andina, quienes los convocan en algunos momentos del proceso formativo. Sin embargo, existen diferentes maneras de concebir el perfil del sabio y su papel en los procesos de formación docente. Mientras que algunos centros de formación optan por conocedores ancianos con los primeros años de educación básica, otros eligen profesores con título profesional. También se observan diferencias en cuanto a la manera como intervienen los sabios en el proceso de formación.

Con relación a este punto, encuentro que mientras algunas instituciones limitan su actuación a la conducción del taller de lengua originaria, otras los invitan a participar en las clases de las diferentes asignaturas o áreas y a dirigir el desarrollo de algunos proyectos, siguiendo el ejemplo de Formabiap, aunque no siempre pueden hacerlo de manera práctica por falta de instalaciones adecuadas $\mathrm{y}$ de recursos que les permitan realizar las actividades productivas.

Un tema aún no resuelto es la falta de inclusión de conocedoras. Si bien esta limitación es difícil de superar debido a las dificultades que las instituciones formadoras encuentran para incorporarlas en procesos de formación docente, que las obligan a dejar a sus familias por largos períodos, es necesario buscar alternativas para asegurar su presencia. De lo contrario se estaría obviando la división sexual del conocimiento, que caracteriza a los pueblos originarios, y se estaría reforzando el valor del conocimiento masculino en desmedro del que ostentan las mujeres.

La institucionalización de la presencia de los conocedores indígenas en los procesos de formación docente es un avance importante. Sin embargo, su impacto en términos de visibilizar conocimientos diferentes a los hegemónicos en la línea de ecología de saberes planteada por Santos (2008) puede quedar trunca si es que no se desarrollan procesos de reflexión crítica sobre el papel que cumplen y podrían cumplir en procesos de construcción de justicia epistémica. De lo contrario, su rol podría quedar limitado al de ser portadores de un conocimiento permanentemente subalternizado dentro de la institución formadora. 


\section{REFERENCIAS BIBLIOGRÁFICAS}

Ávila Romero, Agustín y León Enrique Ávila (2014). El asalto a la interculturalidad: las universidades interculturales de México. Argumentos UAM Xochimilco, 27(76), 37-54.

Fornet Betancourt, Raúl (2001). Filosofía e interculturalidad en América Latina. Intento de introducción no filosófica. Itinerarios, 4, 45-58.

ISPL-Aidesep (1997). Lineamientos curriculares de formación docente en la especialidad de educación primaria intercultural bilingüe. Iquitos.

López Soria, José Ignacio (2012). Universidad e interculturalidad. En F. Tubino y K. Mansilla (eds.), Universidad e interculturalidad. Desafios para América Latina (pp. 13-28). Lima: Pontificia Universidad Católica del Perú.

Mignolo, Walter (2000). Diferencia colonial y razón post occidental. En Castro-Gómez, La reestructuración de las Ciencias Sociales en América Latina (pp. 3-28). Bogotá: Centro Editorial Javeriano.

Nugkuag Evaristo (1985). Experiencia de organización de los pueblos indígenas amazónicos en la actualidad: el Consejo Aguaruna-Huambisa, Amazonas Perú. En J. Gasché y J.M. Arroyo (eds.), Balances amazónicos, enfoques antropológicos (pp. 239-253). Iquitos: CNRS, CIAAP UNAP. Proyecto MAB (UNESCO).

Quijano, Aníbal (2000). Colonialidad del poder, eurocentrismo y América Latina. En Edgardo Lander (comp.), La colonialidad del saber: eurocentrismo y ciencias sociales. Perspectivas Latinoamericanas. Buenos Aires: Clacso.

Santos, Boaventura de Sousa (2008). Una epistemología del sur. México: Siglo XXI.

Trapnell, Lucy (2009). Entre lenguas, entre culturas, proceso de construcción del Programa Formabiap. Sistematización de una experiencia en el Perú. Guatemala: IDIE Organización de Estados Iberoamericanos para la Educación, la Ciencia y la Cultura - OEI.

Tubino, Fidel (2004). Del interculturalismo funcional al interculturalismo crítico. Rostros y fronteras de la identidad, 158. Chile, Universidad Católica de Temuco.

Unesco (2017). Conocimiento indígena y políticas educativas en América Latina. Santiago: OREALC.

Walsh, Catherine (2006). De colonialidad e interculturalidad: reflexiones (des)de proyectos político-epistémicos. En M. Yapu (comp.), Modernidad y pensamiento descolonizador. Memoria Seminario Internacional. La Paz: Fundación PIEB/ Instituto Francés de Estudios Andinos (IFEA). 